\title{
Advanced Adrenocortical Carcinoma - What to do when First-Line Therapy Fails?
}

\author{
Authors \\ Felix Megerle ${ }^{1}$, Matthias Kroiss ${ }^{2}$, Stefanie Hahner ${ }^{1}$, Martin Fassnacht ${ }^{1,2}$
}

\section{Affiliations}

1 Department of Internal Medicine I, Division of Endocrinology and Diabetes, University Hospital Würzburg, University of Würzburg, Germany

2 Comprehensive Cancer Center Mainfranken, University of Würzburg, Germany

Key words

adrenal cancer, ACC, second line therapy

$\begin{array}{lr}\text { received } & 09.07 .2018 \\ \text { revised } & 20.08 .2018 \\ \text { accepted } & 23.08 .2018\end{array}$

\section{Bibliography}

DOI https://doi.org/10.1055/a-0715-1946

Published online: 23.11.2018

Exp Clin Endocrinol Diabetes 2019; 127: 109-116

(C) J. A. Barth Verlag in Georg Thieme Verlag KG Stuttgart .

New York

ISSN 0947-7349

\section{Correspondence}

Martin Fassnacht, MD

University Hospital of Würzburg

Dept. of Internal Medicine I

Division of Endocrinology and Diabetes

Oberdürrbacher Str. 6

97080 Würzburg

Germany

Tel.: +49/931/201 39021, Fax: +49/931/2016039021

fassnacht_m@ukw.de

\begin{abstract}
Adrenocortical carcinoma is a rare endocrine malignant disease with a generally unfavorable but heterogeneous prognosis. Although even in advanced stages a subset of patients experiences long-term disease stabilisation, effective systemic treatment options are limited. Mitotane is the only approved drug and the combination of etoposide, doxorubicin and cisplatin (plus mitotane) is currently considered as treatment standard for advanced adrenocortical carcinoma based on the results of a large randomized phase III trial. However, progression-free survival is often limited and further treatment options are frequently needed. Here we summarize the current knowledge about second and third-line therapeutic modalities (local and systemic) in advanced disease. Following the recent ESE-ENSAT guidelines local therapies play an important role for these patients. Regarding systemic therapies the best data are available for gemcitabine + capecitabine or streptozotocin (both with or without mitotane). Furthermore, we introduce our own approach to patients with advanced adrenocortical carcinoma based on our experience as a large multidisciplinary clinic dedicated to the care of patients with this orphan disease.
\end{abstract}

$\begin{array}{ll}\text { Abbreviations } \\ \text { ACC } & \text { adrenocortical carcinoma } \\ \text { EDP } & \text { etoposide, doxorubicin, cisplatin } \\ \text { EDP-M } & \text { EDP + mitotane } \\ \text { CR } & \text { complete response } \\ \text { PR } & \text { partial response } \\ \text { SD } & \text { stable disease } \\ \text { PFS } & \text { progression-free survival } \\ \text { OS } & \text { overall survival } \\ \text { RFA } & \text { radiofrequency ablation } \\ \text { TACE } & \text { transcatheter arterial chemoembolization } \\ \text { I-MTO } & \text { iod-metomidate }\end{array}$

\section{Introduction}

Adrenocortical carcinoma (ACC) is a rare and aggressive disease with dismal prognosis and limited therapeutic options in advanced tumor stages [1-3]. In patients with metastatic disease 5-year survival rate is below $15 \%$ in most series, but prognosis is heterogeneous [4-8]. We and others have witnessed several patients with multiple metastases and survival of more than 10 years [9]. Very recently the first international guidelines have been published based on an extensive literature search using the GRADE system [3]. According to these recommendations, complete surgical resection is the treatment of choice, if possible. The only drug approved for treatment of ACC is mitotane, which has been in clinical use both in an adjuvant setting 
and for advanced disease for many years [10, 11]. In advanced ACC, mitotane monotherapy is most suitable for patients with less aggressive tumors [3]. Recent data suggest monotherapy with mitotane is particularly suitable for patients with delayed recurrence and limited tumor burden [12]. Objective response rate in this highly selected patients is up to $30 \%$ with a progression-free survival of 8.8 months and an overall survival of 29.6 months. Mitotane is given daily as tablets (Lysodren ${ }^{\circledR}$ ). Therapeutic drug monitoring is mandatory to balance effectivity associated with plasma concentrations $>14 \mathrm{mg} / \mathrm{l}$ and toxicity [13-15].

The only therapy that is based on results of a large randomized phase III trial in advanced stages with irresectable ACC is EDP-M, a combination of etoposide, doxorubicin, cisplatin and mitotane [16]. EDP is administered as an intravenous infusion every 4 weeks mostly in combination with continuous oral mitotane. The FIRM-ACT trial demonstrated an objective response rate in an unselected patient cohort (almost $70 \%$ of patients were already progressive on mitotane monotherapy) of $23 \%$. Additional $35 \%$ of patients experienced stable disease leading to a median progressive-free survival of 5.1 months [16]. The relatively short disease stabilization clearly indicates the important role of second-line therapies as part of disease management in many patients, but data on this topic are scarce. Therefore, it is crucial to adapt the therapeutic concept in advanced ACC for every patient by accounting for individual risk factors, general clinical condition and patient preference. In this review, we summarize therapeutic options for advanced ACC after first-line therapy has failed and we further introduce our current approach to these patients.

\section{Second and Third-Line-Therapies in Advanced ACC}

During the process of a recent guideline development a systematic review of all studies on treatment in advanced ACC since 1986 was performed [3]. The result of this literature search is summarized in > Fig. 1. Of note, all these data have to be interpreted with great caution, because of the different study designs and patient characteristics. While the current ESE-ENSAT guidelines emphasize the important role of local therapies in advanced ACC [3], it has to be pointed out, that the published evidence for the different local options is particularly limited with the lack of any head-to-head comparisons.

\section{Treatments for local tumor control}

Radiofrequency ablation (RFA)

The only study focusing on RFA in ACC demonstrated an objective response in 8 of 15 lesions after percutaneous ablation [17]. Nevertheless, although being the key reference article in this field, the cohort was rather small with only 8 patients included. However, evidence from other diseases as well as our personal experience suggests that RFA is particularly suitable in cases with limited number of liver lesions (e. g. $\leq 3$ ) with a diameter of less than $3 \mathrm{~cm}$.

Transcatheter arterial chemoembolization (TACE)

Data on TACE are also limited. To our knowledge only one study with 29 patients evaluated TACE in advanced ACC [18]. In this series a de- crease in tumor size in $22 \%$ of 103 treated lesions was observed, additional $65 \%$ were stable in size after 3 months. Otherwise, only case reports are available that are often characterized by extraordinary long PFS or OS after TACE (e.g. [19, 20]), which renders reporting bias likely. Although for ACC, there is no established cytotoxic drug to be applied during TACE, most centers use cisplatin, doxorubicin and/or mitomycin C. However, we and others administer this treatment modality sometimes only with lipiodol ${ }^{\circledR}$ (without cytotoxics). Specifically, we use TA(C)E usually in selected patients (e.g. several liver metastases in one lobe of the liver) and continue frequently other systemic therapies (e. g. mitotane and/or cytotoxic chemotherapy), but it can be applied also as single therapy.

\section{Radiation therapy}

ACC has long been considered to be rather resistant to radiation $[21,22]$. More recent data show clinical benefit and objective response after radiation therapy in adjuvant and palliative setting [23-26]. However, except from case series published in reviews (for overview see [25]) no sound investigation of this treatment modality in advanced ACC has yet been performed. As in other cancers, radiotherapy is the treatment of choice for symptomatic bone metastases, which leads to relief of symptoms in 50-90\% of patients [27]. Symptomatic abdominal or brain metastases or metastases, which are expected to become symptomatic frequently require external beam radiation. However, an increasing number of centers (including ours) is applying radiotherapy also in casees of limited disease to achieve local tumor control. Combination with systemic cytotoxic drugs is feasible when respecting patient-individual factors.

\section{Microwave ablation}

Analog to RFA CT-guided percutaneous microwave ablation is a minimally invasive treatment method of solid tumors. Li et al. showed that microwave ablation can be suitable for different adrenal tumors, especially those smaller than $5 \mathrm{~cm}$ in diameter [28]. However, only a single of the included 9 patients had advanced ACC. Again, this option is useful as monotherapy or in combination with other (e.g. systemic) therapies. We personally have no experience with this method.

\section{Systemic therapies}

\section{Streptozotocin (+ /- mitotane)}

Although streptozotocin is approved only in few countries, it has been in use for patients with ACC since the 1980's. With smaller studies showing promising results for this drug in combination with mitotane in adjuvant and therapeutic usage [29, 30], the FIRM-ACT study [16] demonstrated a limited response rate for streptozotocin of $9 \%$ and a median PFS and OS of only 2 months and 12 months, respectively. Nevertheless some patients did show objective tumor response with few complete responders. Although overall disease control rate $(C R+P R+S D)$ was $31.4 \%$, this might in part reflect less aggressive tumor biology in some cases. Of note, PFS and objective response rate in second-line therapy after failing EDP-M was quite similar with 2.1 months and $7.6 \%$, respectively (unpublished data of the FIRM-ACT trial). In our experience, patients did describe the adverse effects of streptozotocin usually as less intense compared to EDP-M, although the rate of serious adverse events did not differ significantly [16]. During the FIRM-ACT trial streptozo- 


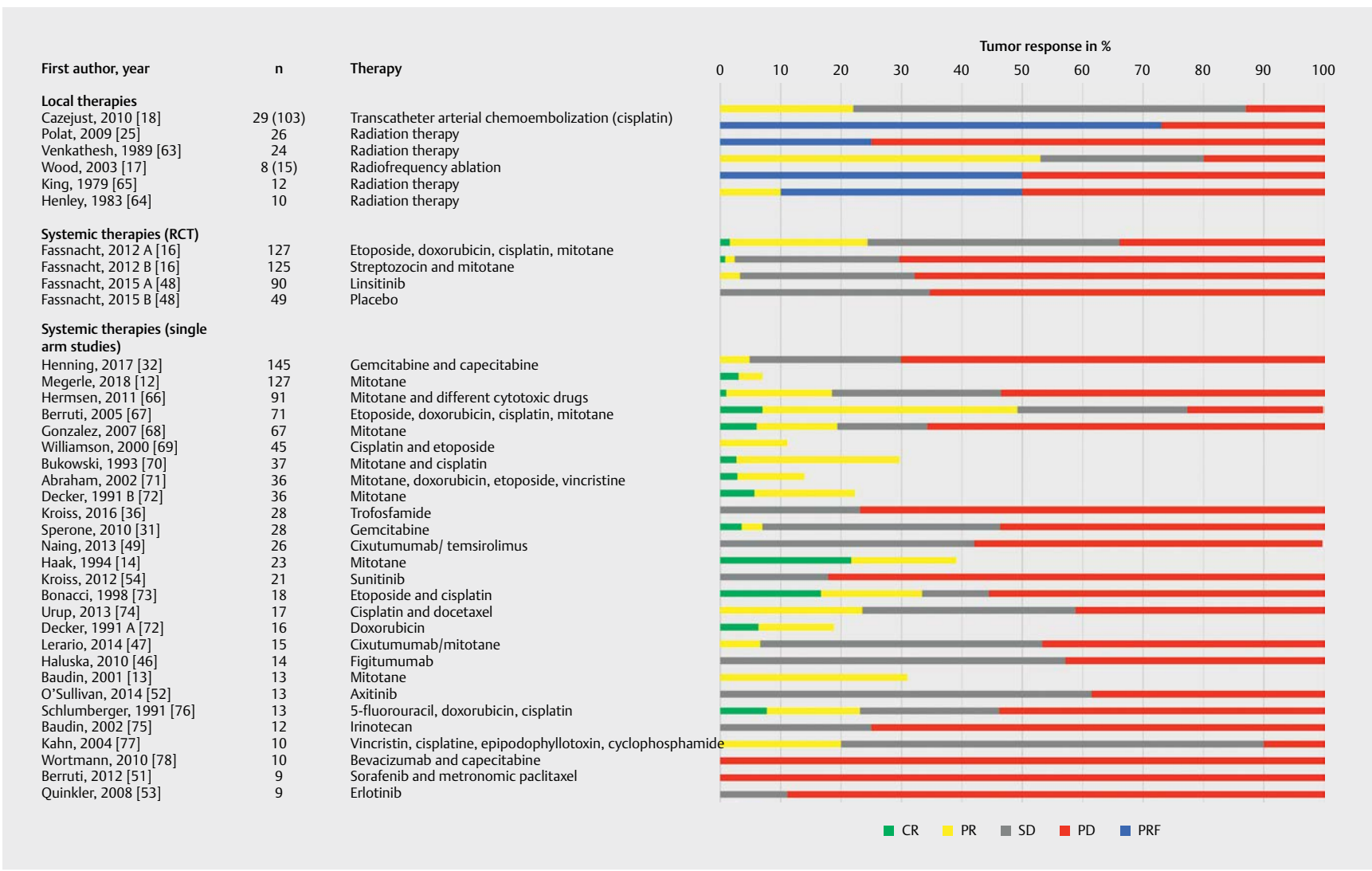

- Fig. 1 Overview of response rates in studies on treatment in advanced ACC since 1986. The upper part of the panel summarizes the local therapies and the lower part the systemic therapies (adapted from Fassnacht 2018[3]) [12-14, 16-18, 25, 31, 32, 36, 46-49, 51-54, 63-78] Ordered by number of included patients per study. Study protocols, patient cohorts and characteristics as well as outcome measurements are quite different so interpretation and comparison is difficult. CR: complete response; PR: partial response; SD: stable disease; PD: progressive disease; PRF: pain relief. Some of the older studies did not report stable disease or progression, thus these columns don't sum up to $100 \%$. In studies on radiation therapy frequently response was defined clinically (e. g. pain relief), which is certainly not comparable with objective response by imaging (these responses are indicated in blue). In two studies response based on the total number of lesions (given in brackets in the second column).

tocin was administered together with mitotane. We have seen benefit of streptozotocin also as monotherapy, but usually combine both drugs. Streptozotocin can safely be combined with radiation therapy, because bone marrow toxicity is very limited. Administration is every 3 weeks as intravenous infusion ( Table $\mathbf{1}$ ).

\section{Gemcitabine and capecitabine (+ /- mitotane)}

A phase 2 clinical trial in patients with advanced ACC $(n=28)$ demonstrated a median PFS of 5.3 months for the combination of gemcitabine and capecitabine with $46 \%$ of patients without progression in the first 4 months [31]. As this trial has been performed exclusively in patients failing at least EDP-M, this combination has been proposed as second-line option in advanced ACC [1]. A larger ( $n=132$ ) retrospective analysis could not completely confirm these results and showed a median PFS only at 3 months with an objective response in $4.9 \%$ of patients and a disease control in $29.9 \%$ of patients with a median duration of 26.8 weeks [32]. Major adverse effects of this regimen include bone marrow suppression, skin rash and hand-foot-syndrome. Gemcitabine is given as intermittent intravenous infusion and capecitabine as continuous oral medication ( $\vee$ Table 1 ) and may be combined with mitotane.

\section{Radionuclide treatment with [131I]-metomidate (I-MTO)}

In 2012, our center introduced the first targeted radionuclide therapy for patients with ACC [33]. The principle of this therapy is based upon the binding of iodo-metomidate to the 11-beta hydroxylase and aldosterone synthase of the normal adrenal gland but also ACC primary tumors and metastases [34]. Radiosynthesis of the 131-I derivative offers the possibility of targeted radionuclide therapy. In a small case series $(n=11)$ clinical benefit (disease control $\geq 5$ months with a median progression free survival of 14 months) could be achieved in $54 \%$ of the patients [33]. Treatment response depends on intratumoral expression of the target enzymes, individual affinity and retention time of the compound in tumor lesions. Highly aggressive tumors may be less susceptible to the effect of radiotherapy. Therefore, only about one third of patients qualifies for treatment [35]. Prospective clinical trials are still lacking.

\section{Trofosfamide}

In a small study $(\mathrm{n}=21)$, we could show disease stabilization with trofosfamid in $14 \%$ of patients with a median PFS of 84 days and median OS of 198 days [36]. No objective response was seen in this cohort of heavily pretreated patients with a median number of three previous regimens. One patient had prolonged disease sta- 
- Table 1 Dosage and administration of ACC therapy.

$1^{\text {st-line therapies }}$

- Surgery $+\mid$ - other local measures

- Mitotane monotherapy

- Starting with high-dose regimen: day $1 \rightarrow 1.5 \mathrm{~g}$, day $2 \rightarrow 3 \mathrm{~g}$, day $3 \rightarrow 4.5 \mathrm{~g}$, day $4 \rightarrow 6 \mathrm{~g}$

- Starting with low-dose regimen: day $1 \rightarrow 1 \mathrm{~g}$, every 3 to 4 days increase by $0.5 \mathrm{~g}$ up to total dose of $3-4 \mathrm{~g} / \mathrm{d}$

- aiming for mitotane blood level $14-20 \mathrm{mg} / \mathrm{L}$, assessment every 3 to 4 weeks (incl. liver enzymes, renal function, blood count)

- key adverse effects: adrenal insufficiency, nausea, diarrhea, cognitive impairment, neurotoxicity, hepatotoxicity, induction of cytochrome p450, elevation of cholesterol and triglycerides

- Etoposide, Doxorubicin and Cisplatin (EDP) plus Mitotane (EDP/M)[12]

- every 28 days: day $1 \rightarrow 40 \mathrm{mg} / \mathrm{m} 2$ doxorubicin $(\mathrm{D})$, day $2 \rightarrow 100 \mathrm{mg} / \mathrm{m} 2$ etoposide $(\mathrm{E})$, day $3+4 \rightarrow 100 \mathrm{mg} / \mathrm{m} 2$ etoposide $(\mathrm{E})+40 \mathrm{mg} / \mathrm{m} 2$ cisplatin $(\mathrm{P})$

- plus oral mitotane aiming at a blood level between $14-20 \mathrm{mg} / \mathrm{l}$

- blood count between day 10 and 14 to adjust dosages in the next cycle

- key adverse effects: nausea, diarrhea, impaired function of bone marrow, hair loss, polyneuropathy/neurotoxicity (P), cardiotoxicity (D)

2nd/3rd-line therapies

- Streptozotocin plus Mitotane (Sz/M)[12]

- induction: day $1-5 \rightarrow 1 \mathrm{~g} \mathrm{Sz} / \mathrm{d}$

- afterwards Sz 2 g/d every 21 days

- plus oral mitotane aiming for mitotane blood level $14-20 \mathrm{mg} / \mathrm{L}$, assessment every 3 to 4 weeks

- blood sampling regularly with liver enzymes, renal function, blood count

- key adverse effects: nausea, diarrhea, rarely impaired function of liver, kidney, impaired function of bone marrow (usually mild)

- Gemcitabine plus Capecitabine[31-32]

- $800 \mathrm{mg} / \mathrm{m}^{2}$ gemcitabine on day 1 and 8 (every 3 weeks)

- $1500 \mathrm{mg}$ capecitabine continuously (oral)

- individual decision about continuing mitotane

- blood sampling regularly with liver enzymes, renal function, blood count

- key adverse effects: nausea, diarrhea, impaired function of bone marrow

Salvage therapies

- Trofosfamide

- max. 150 mg per day in 3 doses

- key adverse effects: impaired function of bone marrow, neurotoxicity, rarely: nephron-/urotoxicity, cardiotoxicity, pulmonary toxicity

\section{- Thalidomide}

- starting dose $50-100 \mathrm{mg} / \mathrm{d}$ (given in a single dose)

- increase (up to $400 \mathrm{mg} / \mathrm{d}$ ) or decrease according to tolerability and side-effects

- adverse effects: impaired function of bone marrow, thromboembolic events, skin rash bradycardia, polyneuropathy/neurotoxicity, cardiotoxicity

bilization for 479 days, which seems to be remarkably in this setting. The drug, which is administered orally was in general very well tolerated with only mild adverse events. Therefore, trofosfamide might be a reasonable salvage therapy in selected patients with reduced clinical conditions, but strong wish to continue antitumor therapy.

\section{Thalidomide}

In 2005, Chacón et al. published a case report of an impressive objective response in advanced ACC with liver metastasis after several months of therapy with thalidomide [37]. Thalidomide is an approved first line treatment for multiple myeloma and acts mainly by direct binding to cereblon protein $[38,39]$ as an immune modulatory drug. In our own experience of 27 cases effectivity is limited and we currently do not advocate the use of thalidomide and related compounds without clarification of response predictors [40].

\section{Targeting the IGF-II/IGF receptor I pathway}

Since many years it is known that IGF-II is the most up-regulated gene in ACC [41-44]. Therefore, it seemed logical to use the IGF receptor signalling pathway as target for therapy. After positive phase I trials [45-47], a large placebo-controlled phase III trial did not hold promise and the IGF1R inhibitor linsitinib was not able to improve progression-free or overall survival [48]. However, long- term partial response in 3 out of 90 patients treated with linsitinib suggests that at least a very small subset of patients might benefit from an IGF-targeting therapy. It is well established that mTOR effects the IGFR signaling pathway and might be useful for treatment of patients with ACC. Recent data suggest that the combination of mTOR-inhibitors and IGF1-receptor antagonists [49] or lenalidomide [50] or other immune modulatory drug, might be an interesting approach. However, we have no personal experience with these combinations yet.

\section{Tyrosine kinase inhibitors}

As in many other solid malignancies, several tyrosine kinase inhibitors (TKI) have been investigated in ACC, but largely with disappointing results [51-53]. The SIRAC-study (Sunitinib In Refractory Adrenocortical (arcinoma) was an eye opening experience that could at least partially explain the perceived lack of efficacy of many TKIs. In this study, sunitinib was given to 35 patients in a standard dosage ( $50 \mathrm{mg} / \mathrm{d}$, 4 weeks on, 2 weeks off) [54]. Stable disease after 12 weeks, the primary end point of this study, was observed in $14.3 \%$ of patients. Median PFS was 83 days and median overall survival 5.4 months. Although these results suggested some potential efficacy of this vascular growth factor receptor 2 directed TKI it became evident, that co-treatment of mitotane was not only not helpful, but probably negatively impacted on the anti-tumoral ef- 
fect of the drug. Only after the study was completed, the fortuitous finding of decreased plasma exposure to sunitinib in mitotanetreated patients was reported and mitotane-induced CYP3A4 induction suggested in two further mitotane treated patients [55]. Accordingly, in the SIRAC trial we retrospectively demonstrated a negative correlation between mitotane plasma concentration and plasma concentrations of sunitinib and its active metabolite. Although prospective demonstration is still lacking and it is unknown which other P450 enzymes are induced by mitotane, based on these and other data [56-58] it is now widely accepted that mitotane-induced CYP3A4 must be taken into account during treatment with mitotane. In fact, more than half of all clinically used drugs - including most TKIs - are metabolized via CYP3A4.

Unfortunately, these insights have not yet resulted in the reconsideration of trials on tyrosine kinase inhibitors in mitotane-naïve patients. However, based on preclinical data, we have used the tyrosine kinase inhibitor cabozantinib, which also targets c-MET in few patients with undetectable mitotane levels. First preliminary results are promising which led to the initiation of two parallel clinical trials in the U.S. (NCT03370718, NCT02867592) and Europe (NCT pending).

\section{Immune checkpoint inhibitors}

In several cancers the advent of immune checkpoint inhibitors targeting e. g. cytotoxic T-lymphocyte antigen 4 (CTLA4), programmed death 1 (PD1) or its ligand PDL-1 has changed the perspective of a significant proportion of patients with several advanced malignant diseases that were previously lacking effective treatment options [59]. However, preliminary data with single immune checkpoint inhibitors were presented at the ASCO congress 2016 showing only modest activity in ACC patients [60]. It appears too early to draw firm conclusions about efficacy of immune checkpoint inhibitors in general, however evidence from other tumors suggests that it might be worthwhile to pre-select patients (e. g. tumors that express PD1/PD-L1 or tumors with high mutational load). Several clinical trials (NCT02673333, Pembrolizumab; NCT02834013 and NCT03333616, Nivolumab and Ipilimumab) are currently ongoing that will broaden our knowledge about single and combinatorial treatment regimens in ACC.

\section{How to deal with mitotane in late stage ACC}

Mitotane has been considered as a cornerstone of any medical ACC therapy for decades. However, in our view the significant burden caused by its plethora of adverse effects and its potential of drug interactions in our view should justify the critical reappraisal of treatment indication at regular intervals (e.g. every restaging) during the course of ACC. The panelists of the ESE-ENSAT guideline could not come to a consensus on the best time point, when to discontinue mitotane [3]. In patients with severe hormone excess continuation of mitotane might be considered, but even in these cases the administration of other inhibitors of the steroidogenesis like metyrapone or ketoconazole may come with lesser toxicity. Our current approach is to consider the individual tolerability of mitotane, dose requirement, tolerability of chemotherapy, cortisol excess and attainment of the therapeutic plasma concentration of mitotane (>14 mg/l). Therefore, we suggest most patients to eventually stop mitotane at the time of a second disease progression on a systemic treatment beyond mitotane monotherapy. Others suggest to discontinue mitotane in any patient who experiences progressive disease one year after starting mitotane [61].

\section{The Current "Würzburg Approach" (Fig. 2) and Future Perspectives}

Treatment of patients with advanced ACC refractory to "standard therapy" is always challenging. At the latest after failing systematic therapy with EDP-M, we strongly encourage to discuss with the patient once more individually the prognosis and preferences of the patient. Sometimes, the most appropriate approach is to stop active antitumor therapy and to provide best supportive care following the tradition of Hippocrates "primum nil nocere". However, it is our experience that many (often young) ACC patients desire active therapy and are willing to clutch at any straw. To offer these patients - after a transparent informed consent process - therapeutic options that are based on published trials or evidence from preclinical studies has frequently quite positive effects on their quality of life. The fact that we have seen single patients who benefited clearly from different salvage therapies in our view justifies this concept.

In our current approach we address first the following three questions: (i) Is there a suitable clinical phase I or II trial in our center (or anywhere else within reach of the patient) that is currently or will be within a short time interval recruiting and likely to include the patient? (ii) Is there an option to administer any local therapy to achieve tumor control? (iii) Is the tumor burden and dynamics of tumor growth limited and is there sufficient uptake of a metomidate-based tracer to allow radionuclide therapy? If all questions are answered with no, we usually suggest either streptozotocin ( \pm mitotane) or gemcitabine + capecitabine ( \pm mitotane). Often a combination of more than one therapy (e. g. radiation + systemic chemotherapy) is necessary to provide patients with the best approach for their individual course of disease. If the disease is further progressing after these two options, we again discuss the values of a best-supportive care concept (ideally close to the patient's home). Latest at that point we usually discontinue mitotane therapy (see above for details) and consider to include the patient in one of the available programs for personalized medicine [62], potentially including the biopsy of a suitable tumor lesion which might potentially result in compassionate use treatment.

Evidently, there is no standard operating procedure, which can be extrapolated to fit every patient. The process will always reflect an individual shared decision making of the patient and the treating physician. Potential options are given in $\mathbf{F i g .} 2$ and details are provided in the text above and $>$ Table 1.

In general, during therapy for advanced ACC, we recommend to evaluate treatment response by cross-sectional imaging of the abdomen, pelvis and thorax every 8-12 weeks [3]. The best imaging interval depends on the dynamics of the disease, disease symptoms including hormone excess or likelihood of symptomatic disease, toxicity and cost of the ongoing treatment, and the overall prognosis of the patient. In patients with best supportive care we recommend to discontinue systematic imaging.

In view of current initiatives to re-evaluate the utility of small molecules within clinical drug trials or personalized medicine programs, 


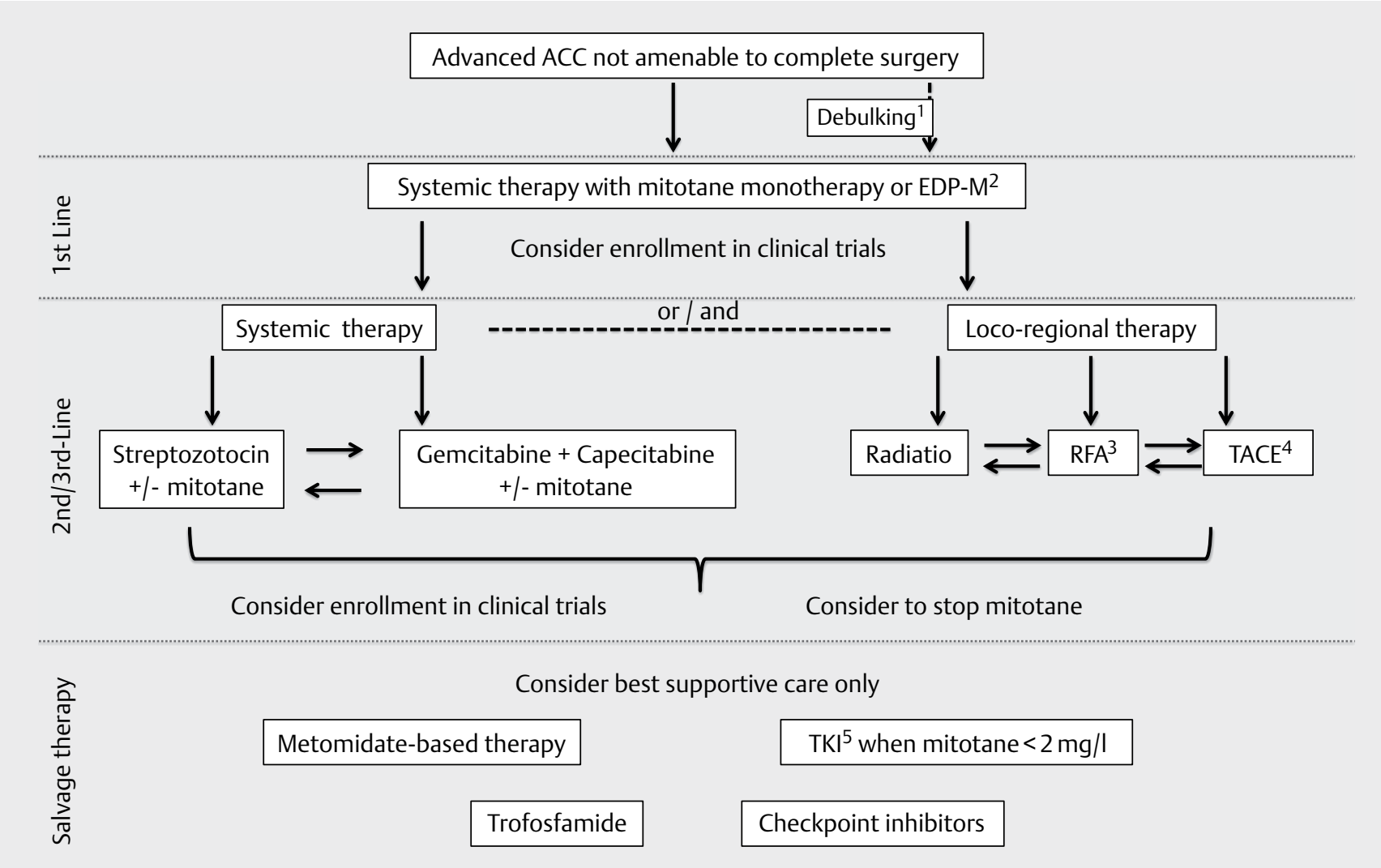

Fig. 2 FlowChart on the management of patients with advanced ACC. ${ }^{1}$ In case of hormonal excess (e. g. Cushing syndrome), which can not be controlled otherwise. ${ }^{2}$ EDP-M : etoposide + doxorubicin + cisplatin + mitotane. ${ }^{3}$ RFA: radiofequency ablation. ${ }^{4}$ TACE: transcatheter arterial chemoembolization. ${ }^{5} \mathrm{TKI}$ : tyrosine kinase inhibitor.

we focus on the individual requirement of mitotane. Improvement of patient selection, improvements of radiochemistry and choice of dose in iodometomidate treatment is another promising line of clinical research. Immunotherapy probably will require improved patient selection or combination therapy. On the long-term the best would be if we can prevent that patients develop advanced ACC at all. In this context, the currently ongoing and initiated trials on adjuvant therapy (ADIUVO, NCT00777244 and ADIUVO II, NCT03370718) will hopefully help to achieve this goal. Therefore, we can only encourage all treating physicians and patients to participate in clinical trial as the best way to improve our knowledge in ACC.

Finally, there is no doubt that an improved understanding of the pathophysiology of ACC is required to pave the way to a significant better treatment of our patients. Therefore, international networks focusing on preclinical and clinical studies, like the European Network of the Study of Adrenal Tumors, ENS@T) are crucial, because only combined efforts of many expert centers will be able to overcome the hurdles of a very rare disease.

\section{Funding}

This study was supported in part by the Deutsche Forschungsgemeinschaft (DFG) within the CRC/Transregio 205/1 „The Adrenal: Central Relay in Health and Disease“ (project B16) to M.K. and M.F. and an individual research grant to M.K. (KR 4371/1-2) and M.F. (FA466/4-2).

\section{Conflict of Interest}

No conflict of interest has been declared by the authors.

\section{References}

[1] Berruti A, Baudin E, Gelderblom H et al. Adrenal cancer: ESMO clinical practice guidelines for diagnosis, treatment and follow-up. Ann Oncol 2012; 23: (Suppl 7): vii131-vii138

[2] Else T, Kim AC, Sabolch A et al. Adrenocortical carcinoma. Endocr Rev 2014; 35: 282-326

[3] Fassnacht M, Dekkers OM, Else T et al. Management of adrenocortical carcinoma in adult patients - A European Society of Endocrinology Clinical Practice Guideline in collaboration with the European Network for the Study of Adrenal Tumors. Eur J Endocrinol 2018, 2018 Jul 24. pii: EJE-18-0608. doi:10.1530/EJE-18-0608

[4] Abiven G, Coste J, Groussin L et al. Clinical and biological features in the prognosis of adrenocortical cancer: poor outcome of cortisolsecreting tumors in a series of 202 consecutive patients. J Clin Endocrinol Metab 2006; 91: 2650-2655

[5] Assie G, Antoni G, Tissier F et al. Prognostic parameters of metastatic adrenocortical carcinoma. J Clin Endocrinol Metab 2007; 92: 148-154

[6] Fassnacht M, Allolio B. Clinical management of adrenocortical carcinoma. Best Pract Res Clin Endocrinol Metab 2009; 23: 273-289 
[7] Icard P, Goudet P, Charpenay C et al. Adrenocortical carcinomas: Surgical trends and results of a 253-patient series from the French Association of Endocrine Surgeons study group. World J Surg 2001; 25: 891-897

[8] Libe R. Adrenocortical carcinoma (ACC): Diagnosis, prognosis, and treatment. Front Cell Dev Biol 2015; 3: 45

[9] Hermsen IG, Gelderblom H, Kievit J et al. Extremely long survival in six patients despite recurrent and metastatic adrenal carcinoma. Eur J Endocrinol 2008; 158: 911-919

[10] Schteingart DE, Doherty GM, Gauger PG et al. Management of patients with adrenal cancer: recommendations of an international consensus conference. Endocr Relat Cancer 2005; 12: 667-680

[11] Terzolo M, Angeli A, Fassnacht M et al. Adjuvant mitotane treatment for adrenocortical carcinoma. N Engl J Med 2007; 356: 2372-2380

[12] Megerle F, Herrmann W, Schloetelburg W et al. Mitotane monotherapy in patients with advanced adrenocortical carcinoma. J Clin Endocrinol Metab 2018, doi:10.1210/jc.2017-02591

[13] Baudin E, Pellegriti G, Bonnay M et al. Impact of monitoring plasma 1,1-dichlorodiphenildichloroethane (o,p'DDD) levels on the treatment of patients with adrenocortical carcinoma. Cancer 2001; 92: 1385-1392

[14] Haak HR, Hermans J, van de Velde C] et al. Optimal treatment of adrenocortical carcinoma with mitotane: results in a consecutive series of 96 patients. $\mathrm{Br}$ J Cancer 1994; 69: 947-951

[15] van Slooten H, Moolenaar AJ, van Seters AP et al. The treatment of adrenocortical carcinoma with o,p'-DDD: Prognostic implications of serum level monitoring. Eur J Cancer Clin Oncol 1984; 20: 47-53

[16] Fassnacht M, Terzolo M, Allolio B et al. Combination chemotherapy in advanced adrenocortical carcinoma. N Engl J Med 2012; 366: 2189-2197

[17] Wood B], Abraham J, Hvizda JL et al. Radiofrequency ablation of adrenal tumors and adrenocortical carcinoma metastases. Cancer 2003; 97: 554-560

[18] Cazejust J, De Baere T, Auperin A et al. Transcatheter arterial chemoembolization for liver metastases in patients with adrenocortical carcinoma. J Vasc Interv Radiol 2010; 21: 1527-1532

[19] Wong $E$, Jacques $S$, Bennett $M$ et al. Complete response in a patient with stage IV adrenocortical carcinoma treated with adjuvant trans-catheter arterial chemo-embolization (TACE). Asia Pac J Clin Oncol 2017. doi:10.1111/ajco.12759

[20] Li SH, Huang CH, Ko SF et al. Extended survival in a patient with recurrent and metastatic adrenal cortical carcinoma by aggressive transarterial embolization-a case report. J Surg Oncol 2005; 90: 101-105

[21] Luton JP, Cerdas S, Billaud L et al. Clinical features of adrenocortical carcinoma, prognostic factors, and the effect of mitotane therapy. N Engl J Med 1990; 322: 1195-1201

[22] Pommier RF, Brennan MF. An eleven-year experience with adrenocortical carcinoma. Surgery 1992; 112: 963-970 discussion 970-961

[23] Fassnacht M, Hahner S, Polat B et al. Efficacy of adjuvant radiotherapy of the tumor bed on local recurrence of adrenocortical carcinoma. J Clin Endocrinol Metab 2006; 91: 4501-4504

[24] Ho J, Turkbey B, Edgerly M et al. Role of radiotherapy in adrenocortical carcinoma. Cancer J 2013; 19: 288-294

[25] Polat B, Fassnacht M, Pfreundner $L$ et al. Radiotherapy in adrenocortical carcinoma. Cancer 2009; 115: 2816-2823

[26] Sabolch A, Feng M, Griffith K et al. Adjuvant and definitive radiotherapy for adrenocortical carcinoma. Int J Radiat Oncol Biol Phys 2011; 80 : 1477-1484

[27] Pin Y, Paix A, Le Fevre $C$ et al. A systematic review of palliative bone radiotherapy based on pain relief and retreatment rates. Crit Rev Oncol Hematol 2018; 123: 132-137
[28] Li X, Fan W, Zhang L et al. CT-guided percutaneous microwave ablation of adrenal malignant carcinoma: Preliminary results. Cancer 2011; 117: 5182-5188

[29] Eriksson B, Oberg K, Curstedt T et al. Treatment of hormone-producing adrenocortical cancer with o,p'DDD and streptozocin. Cancer 1987; 59: 1398-1403

[30] Khan TS, Imam H, Juhlin C et al. Streptozocin and o,p'DDD in the treatment of adrenocortical cancer patients: Long-term survival in its adjuvant use. Ann Oncol 2000; 11: 1281-1287

[31] Sperone P, Ferrero A, Daffara F et al. Gemcitabine plus metronomic 5-fluorouracil or capecitabine as a second-/third-line chemotherapy in advanced adrenocortical carcinoma: A multicenter phase II study. Endocr Relat Cancer 2010; 17: 445-453

[32] Henning JEK, Deutschbein T, Altieri B et al. Gemcitabine-based chemotherapy in adrenocortical carcinoma: a multicentric study on efficacy and predictive factors. J Clin Endocrinol Metab 2017. jc.2017-01624-jc.02017-01624

[33] Hahner S, KreissI MC, Fassnacht M et al. [1311]iodometomidate for targeted radionuclide therapy of advanced adrenocortical carcinoma. J Clin Endocrinol Metab 2012; 97: 914-922

[34] Hahner S, Sundin A. Metomidate-based imaging of adrenal masses. Horm Cancer 2011; 2: 348-353

[35] Kreissl MC, Schirbel A, Fassnacht M et al. [(1)(2)(3)!]lodometomidate imaging in adrenocortical carcinoma. J Clin Endocrinol Metab 2013; 98: $2755-2764$

[36] Kroiss M, Deutschbein T, Schlotelburg W et al. Salvage treatment of adrenocortical carcinoma with trofosfamide. Horm Cancer 2016; 7: 211-218

[37] Chacon R, Tossen G, Loria FS et al. CASE 2. Response in a patient with metastatic adrenal cortical carcinoma with thalidomide. J Clin Oncol 2005; 23: 1579-1580

[38] Lu G, Middleton RE, Sun $\mathrm{H}$ et al. The myeloma drug lenalidomide promotes the cereblon-dependent destruction of Ikaros proteins. Science 2014; 343: 305-309

[39] Zhu YX, Braggio E, Shi CX et al. Cereblon expression is required for the antimyeloma activity of lenalidomide and pomalidomide. Blood 2011; 118: 4771-4779

[40] Kroiss M, Deutschbein T, Schloetelburg W. Treatment of refractory adrenocortical carcinoma with thalidomide: Analysis of 27 patients from the European Network for the Study of Adrenal Tumours Registry. Exp Clin Endocrinol Diabetes 2018 doi:10.1055/a-0747-5571

[41] de Reynies A, Assie G, Rickman DS et al. Gene expression profiling reveals a new classification of adrenocortical tumors and identifies molecular predictors of malignancy and survival. J Clin Oncol 2009; 27: $1108-1115$

[42] Gicquel C, Bertagna X, Gaston V et al. Molecular markers and long-term recurrences in a large cohort of patients with sporadic adrenocortical tumors. Cancer Res 2001; 61: 6762-6767

[43] Gicquel C, Bertagna X, Schneid H et al. Rearrangements at the 11p15 locus and overexpression of insulin-like growth factor-II gene in sporadic adrenocortical tumors. J Clin Endocrinol Metab 1994; 78: 1444-1453

[44] Gicquel C, Raffin-Sanson ML, Gaston V et al. Structural and functional abnormalities at $11 \mathrm{p} 15$ are associated with the malignant phenotype in sporadic adrenocortical tumors: study on a series of 82 tumors. J Clin Endocrinol Metab 1997; 82: 2559-2565

[45] Barlaskar FM, Spalding AC, Heaton JH et al. Preclinical targeting of the type I insulin-like growth factor receptor in adrenocortical carcinoma. J Clin Endocrinol Metab 2009; 94: 204-212 
[46] Haluska P, Worden F, Olmos D et al. Safety, tolerability, and pharmacokinetics of the anti-IGF-1R monoclonal antibody figitumumab in patients with refractory adrenocortical carcinoma. Cancer Chemother Pharmacol 2010; 65: 765-773

[47] Lerario AM, Worden FP, Ramm CA et al. The combination of insulin-like growth factor receptor 1 (IGF1R) antibody cixutumumab and mitotane as a first-line therapy for patients with recurrent/metastatic adrenocortical carcinoma: a multi-institutional $\mathrm{NCl}$-sponsored trial. Horm Cancer 2014; 5: 232-239

[48] Fassnacht M, Berruti A, Baudin E et al. Linsitinib (OSI-906) versus placebo for patients with locally advanced or metastatic adrenocortical carcinoma: a double-blind, randomised, phase 3 study. Lancet Oncol 2015; 16: 426-435

[49] Naing A, Lorusso P, Fu S et al. Insulin growth factor receptor (IGF-1R) antibody cixutumumab combined with the mTOR inhibitor temsirolimus in patients with metastatic adrenocortical carcinoma. Br J Cancer 2013; 108: 826-830

[50] Ganesan P, Piha-Paul S, Naing A et al. Phase I clinical trial of lenalidomide in combination with temsirolimus in patients with advanced cancer. Invest New Drugs 2013; 31: 1505-1513

[51] Berruti A, Sperone P, Ferrero A et al. Phase II study of weekly paclitaxel and sorafenib as second/third-line therapy in patients with adrenocortical carcinoma. Eur J Endocrinol 2012; 166: 451-458

[52] O'Sullivan C, Edgerly M, Velarde M et al. The VEGF inhibitor axitinib has limited effectiveness as a therapy for adrenocortical cancer. J Clin Endocrinol Metab 2014; 99: 1291-1297

[53] Quinkler M, Hahner S, Wortmann S et al. Treatment of advanced adrenocortical carcinoma with erlotinib plus gemcitabine. J Clin Endocrinol Metab 2008; 93: 2057-2062

[54] Kroiss M, Quinkler M, Johanssen S et al. Sunitinib in refractory adrenocortical carcinoma: a phase II, single-arm, open-label trial. J Clin Endocrinol Metab 2012; 97: 3495-3503

[55] van Erp NP, Guchelaar HJ, Ploeger BA et al. Mitotane has a strong and a durable inducing effect on CYP3A4 activity. Eur J Endocrinol 2011; 164: 621-626

[56] Chortis V, Taylor AE, Schneider P et al. Mitotane therapy in adrenocortical cancer induces CYP3A4 and inhibits 5alpha-reductase, explaining the need for personalized glucocorticoid and androgen replacement. J Clin Endocrinol Metab 2013; 98: 161-171

[57] Kroiss M, Quinkler M, Lutz WK et al. Drug interactions with mitotane by induction of CYP3A4 metabolism in the clinical management of adrenocortical carcinoma. Clin Endocrinol 2011; 75: 585-591

[58] Theile D, Haefeli WE, Weiss J. Effects of adrenolytic mitotane on drug elimination pathways assessed in vitro. Endocrine 2015; 49: 842-853

[59] Topalian SL, Drake CG, Pardoll DM. Immune checkpoint blockade: A common denominator approach to cancer therapy. Cancer Cell 2015; 27: $450-461$

[60] Le Tourneau C, Hoimes C], Zarwan C et al. Avelumab (MSB0010718C; anti-PD-L1) in patients with advanced adrenocortical carcinoma from the JAVELIN solid tumor phase Ib trial: Safety and clinical activity. J Clin Oncol 2016; 34

[61] Vezzosi D, Do Cao C, Hescot S et al. Time until partial response in metastatic adrenocortical carcinoma long-term survivors. Horm Cancer 2017, doi:10.1007/s12672-017-0313-6
[62] Lippert ], Appenzeller S, Liang R et al. Targeted molecular analysis in adrenocortical carcinomas: A strategy towards improved personalized prognostication. J Clin Endocrinol Metab 2018. doi:10.1210/ jc.2018-01348

[63] Venkatesh S, Hickey RC, Sellin RV et al. Adrenal cortical carcinoma. Cancer 1989; 64: 765-769

[64] Henley DJ, van Heerden JA, Grant CS et al. Adrenal cortical carcinomaA continuing challenge. Surgery 1983; 94: 926-931

[65] King DR, Lack EE. Adrenal cortical carcinoma: A clinical and pathologic study of 49 cases. Cancer 1979; 44: 239-244

[66] Hermsen IG, Fassnacht M, Terzolo M et al. Plasma concentrations of o,p'DDD, o,p'DDA, and o,p'DDE as predictors of tumor response to mitotane in adrenocortical carcinoma: Results of a retrospective ENS@T multicenter study. J Clin Endocrinol Metab 2011; 96: 1844-1851

[67] Berruti A, Terzolo M, Sperone P et al. Etoposide, doxorubicin and cisplatin plus mitotane in the treatment of advanced adrenocortical carcinoma: A large prospective phase II trial. Endocr Relat Cancer 2005; 12: 657-666

[68] Gonzalez RJ, Tamm EP, $\mathrm{Ng} \mathrm{C} \mathrm{et} \mathrm{al.} \mathrm{Response} \mathrm{to} \mathrm{mitotane} \mathrm{predicts}$ outcome in patients with recurrent adrenal cortical carcinoma. Surgery 2007; 142: 867-875. discussion 867-875

[69] Williamson SK, Lew D, Miller G] et al. Phase II evaluation of cisplatin and etoposide followed by mitotane at disease progression in patients with locally advanced or metastatic adrenocortical carcinoma: A Southwest Oncology Group Study. Cancer 2000; 88: 1159-1165

[70] Bukowski RM, Wolfe M, Levine HS et al. Phase II trial of mitotane and cisplatin in patients with adrenal carcinoma: A Southwest Oncology Group study. J Clin Oncol 1993; 11: 161-165

[71] Abraham J, Bakke S, Rutt A et al. A phase II trial of combination chemotherapy and surgical resection for the treatment of metastatic adrenocortical carcinoma: continuous infusion doxorubicin, vincristine, and etoposide with daily mitotane as a P-glycoprotein antagonist. Cancer 2002; 94: 2333-2343

[72] Decker RA, Elson P, Hogan TF et al. Eastern Cooperative Oncology Group study 1879: Mitotane and adriamycin in patients with advanced adrenocortical carcinoma. Surgery 1991; 110: 1006-1013

[73] Bonacci R, Gigliotti A, Baudin E et al. Cytotoxic therapy with etoposide and cisplatin in advanced adrenocortical carcinoma. $\mathrm{Br}$ J Cancer 1998; 78: 546-549

[74] Urup T, Pawlak WZ, Petersen PM et al. Treatment with docetaxel and cisplatin in advanced adrenocortical carcinoma, a phase II study. $\mathrm{Br}$ J Cancer 2013; 108: 1994-1997

[75] Baudin E, Docao C, Gicquel C et al. Use of a topoisomerase I inhibitor (irinotecan, CPT-11) in metastatic adrenocortical carcinoma. Ann Oncol 2002; 13: 1806-1809

[76] Schlumberger M, Brugieres L, Gicquel C et al. 5-Fluorouracil, doxorubicin, and cisplatin as treatment for adrenal cortical carcinoma. Cancer 1991; 67: 2997-3000

[77] Khan TS, Sundin A, Juhlin C et al. Vincristine, cisplatin, teniposide, and cyclophosphamide combination in the treatment of recurrent or metastatic adrenocortical cancer. Med Oncol 2004; 21: 167-177

[78] Wortmann S, Quinkler M, Ritter C et al. Bevacizumab plus capecitabine as a salvage therapy in advanced adrenocortical carcinoma. Eur ] Endocrinol 2010; 162: 349-356 